\title{
Etude des caractéristiques structurales des teckeraies en fonction de l'âge et du type de sol au Sud et au Centre Bénin
}

\author{
Denis GNANGUENON-GUESSE*, Germain S. NOUNAGNON, \\ Augustin Kossi N. AOUDJI et Jean Cossi GANGLO \\ Université d'Abomey-Calavi, Faculté des Sciences Agronomiques, Laboratoire des Sciences Forestières. \\ BP: 1493 Calavi, Bénin. \\ *Auteur correspondant,E-mail: guessedenis@gmail.com
}

\section{RÉSUMÉ}

La présente étude conduite dans les plantations de teck du Sud et du Centre Bénin a pour objectif de caractériser leur structure en termes de paramètres dendrométriques et de distribution en classes de diamètres en fonction de l'âge et du type de sol. Sur la base d'un dispositif d'échantillonnage systématique à maille carrée de $100 \mathrm{~m}$ x $100 \mathrm{~m}$, les plantations de teck âgées de 5, 10, 15, 25 et 35 ans évoluant sur différents types de sol ont été inventoriées. Les unités d'inventaire sont des placettes de $20 \mathrm{~m}$ x $15 \mathrm{~m}$ pour les peuplements d'âge inférieur à 15 ans et $30 \mathrm{~m}$ x $30 \mathrm{~m}$ pour les peuplements âgés d'au moins 15 ans. D'après les principaux résultats, les valeurs moyennes des densités varient de 114 à 1107 arbres/ha; le diamètre de 6,03 à $39,12 \mathrm{~cm}$; la hauteur moyenne de 5,8 à 23,47 m; la hauteur dominante de 7,01 à $23,71 \mathrm{~m}$; la surface terrière de 3,23 à 16,83 $\mathrm{m}^{2} /$ ha et le facteur d'espacement de 23,81 à $50,95 \%$. Sur le vertisol, on note les meilleures performances sylvicoles. L'âge et le sol influencent globalement les paramètres dendrométriques et les structures des peuplements.

(C) 2017 International Formulae Group. All rights reserved.

Mots clés: Caractéristiques structurales, Tectona grandis, type de sol, âge, sud et centre Bénin

\section{Structural characteristics of teak plantations according to their age and the soil type in Southern and Central Benin}

\begin{abstract}
This research was conducted in teak plantations in Southern and Central Benin, in order to characterize the structure of their dendrometric parameters and diameter class distribution according to their age and soil type. On the basis of a systematic sampling system with a square mesh of $100 \mathrm{~m} \times 100 \mathrm{~m}$, plantations aged of $5,10,15,25$ and 35 on different types of soil were inventoried through $20 \mathrm{mx} 15 \mathrm{~m}$ plots for stands of less than 15 years old, and $30 \mathrm{~m} \mathrm{x} 30 \mathrm{~m}$ for 15 years and older stands. According to the main results, the average values of the densities range from 114 to 1107 trees / ha, the diameter from 6.03 to $39.12 \mathrm{~cm}$. The average height was from 5.8 to $23.47 \mathrm{~m}$ while the dominant height ranged from 7.01 to $23.71 \mathrm{~m}$; the basal area was from 3.23 to
\end{abstract}


$16.83 \mathrm{~m}^{2} /$ ha and the spacing factor from 23.81 to $50.95 \%$. The best silvicultural performances were noticed on vertisol. We conclude that age and type of soil have effect on stands parameters and structures.

(C) 2017 International Formulae Group. All rights reserved.

Keywords: Structural characteristics, Tectona grandis, soil type, age, Southern and Central Benin.

\section{INTRODUCTION}

La gestion durable des forêts repose en partie sur notre capacité à prédire l'évolution des différents peuplements, en termes de composition, de structure, de production et de qualité du bois, en fonction des conditions stationnelles et de différents scénarios sylvicoles (Hitimana et al., 2004; Goreaud et al., 2007). Les contraintes à la gestion durable des forêts en Afrique Subsaharienne sont entre autres la connaissance limitée de l'état des forêts et de leurs conditions et fonctions en termes de structure, composition, régénération et l'évaluation quantitative des bénéfices tangibles et intangibles qu'on peut en tirer (Hitimana et al., 2004).

Le Bénin, depuis 1949, à travers plusieurs projets et programmes de reboisement, a installé de nos jours plus de 20000 ha de plantations forestières domaniales (ONAB, 2005; ONAB, 2014). Parmi les essences utilisées pour ces plantations, le teck (Tectona grandis L.f) est de loin la plus importante en raison de son fort potentiel de croissance et de la grande qualité de son bois (Azankpan, 2002; Anoumou, 2007). Le teck a un potentiel économique important (Parde, 2002). Il est l'une des espèces tropicales ligneuses les plus importantes du marché international du bois de grande qualité. Sa grande durabilité, sa bonne stabilité dimensionnelle et ses qualités esthétiques en font une espèce très précieuse pour la sylviculture des plantations. Les taux de croissance et la forte demande de son bois dur pour la navigation, la construction et l'ameublement font du teck, une option très profitable pour les programmes forestiers publics comme privés (Bermejoa et al., 2003).

C'est pour lever les contraintes liées à la gestion durable de ces plantations, que la présente étude s'est fixé pour objectif de caractériser la structure en termes de paramètres dendrométriques et de distribution par classes de diamètre des teckeraies en vue de contribuer à leur aménagement. La structure d'un peuplement forestier est l'allure, la forme, le mode d'arrangement de ce peuplement (Akouèhou et Ayelo, 2005; Colin et Lambert, 2012; Koura et al., 2013; Gbesso et al., 2013). Les paramètres dendrométriques en termes de densité, diamètre de l'arbre de surface terrière moyenne, la surface terrière, la hauteur moyenne, la hauteur dominante et le facteur d'espacement sont des données chiffrées pour affiner la description et mieux comprendre l'évolution d'une parcelle (Massenet, 2010). Selon le même auteur, ils constituent des indicateurs précieux de la productivité dont on dispose et aussi, de l'état sylvicole des plantations, gage d'une bonne planification des interventions sylvicoles dont les éclaircies. La distribution par classes de diamètre renseigne sur la répartition en petits bois (PB), bois moyens (BM) et gros bois (GB), voire très gros bois (TGB) (Massenet, 2010). Elle varie en fonction des facteurs du milieu tels que les conditions environnementales, les conditions climatiques, les processus de régénération et la concurrence pour les ressources (HerreroJáuregui et al., 2012). Sa connaissance est indispensable pour renseigner sur la structure de l'espèce, les contraintes sylvicoles éventuelles et l'état des teckeraies.

D'importants travaux de recherche ont été réalisés sur les teckeraies domaniales du Bénin. Ils ont permis d'identifier les stations forestières, de caractériser la structure et la dynamique des peuplements et de proposer des interventions sylvicoles et des aménagements appropriés (Ganglo et De Foucault, 2006; Sèwadé, 2010; Bello, 2015; Kpadonou, 2015). La présente étude s'inscrit dans la poursuite des efforts antérieurs, et a pour objectif de décrire les paramètres dendrométriques et les structures en diamètre 
des plantations de teck en fonction de l'âge et du type de sol. L'étude du regroupement des tiges par catégories de grosseur présente un grand intérêt en matière de gestion forestière car elle est une expression de la structure du peuplement (Colin et Lambert, 2012; Koura et al., 2013). Cela permettra de proposer des mesures d'aménagement et de gestion durables des teckeraies du Sud et Centre Bénin. Trois hypothèses ont guidé nos investigations (i) les caractéristiques structurales des plantations de teck varient en fonction $\mathrm{du}$ type de sol; (ii) les caractéristiques structurales des plantations sont identiques quel que soit l'âge et la troisième présume que le type de sol et l'âge influencent simultanément les caractéristiques structurales des plantations de teck du Sud et Centre Bénin.

\section{MATÉRIEL ET MÉTHODES Milieu d'étude}

La présente étude a été conduite dans les plantations de teck du Sud et du Centre Bénin, dans les secteurs forestiers d'Agrimey, de Djigbé, de Toffo et de Massi (Figure 1) qui couvrent une superficie d'environ 10000 ha (ONAB, 2005). Elles sont installées à partir de l'année 1949 (Ganglo et al., 1999). Comprises entre les latitudes $6^{\circ} 49^{\prime}$ et $7^{\circ} 4^{\prime}$ Nord et les longitudes $2^{\circ} 05^{\prime}$ et $2^{\circ} 22^{\prime}$ Est, elles se trouvent à cheval sur le département de l'Atlantique et le département du Zou. Ces plantations bénéficient d'un climat de type subéquatorial en transition vers les climats tropicaux (soudanien ou soudano-guinéen) caractérisé par une pluviométrie moyenne annuelle variant de $1100 \mathrm{~mm}$ à $1200 \mathrm{~mm}$ (ONAB, 2005). Elles sont toutes caractérisées par quatre saisons: deux saisons pluvieuses alternant avec deux saisons sèches. La température moyenne varie de $27^{\circ} \mathrm{C}$ à $28^{\circ} \mathrm{C}$ (ONAB, 2005).

\section{Collecte des données}

L'étude a été menée sur les sousparcelles âgées de 5, 10, 15, 25 et 35 ans dans les quatre secteurs forestiers (Agrimey, Djigbé, Toffo et Massi) et sur les trois types de sols: le vertisol, les sols ferrugineux et les sols ferralitiques. En effet, 15 parcelles ont été sélectionnées à raison de cinq par type de sol. Pour le sol ferrugineux, il y a 04 sousparcelles à Agrimey (12b, 28a, 13c et 25d) et une sous-parcelle à Djigbé $(37 \mathrm{a})$. Cinq sousparcelles (41a, 29a, 22a, 5d, 23b) ont été sélectionnées sur le sol ferralitique, dans le secteur de Djigbé. Sur le vertisol, quatre sousparcelles ont été choisies à Toffo $(9 \mathrm{a}, 1 \mathrm{a}, 1 \mathrm{c}$, 2c) et une dans le secteur forestier de Massi (26a). Chaque sous-parcelle retenue a été inventoriée sur la base d'un dispositif d'échantillonnage systématique à maille carrée de 100 m x 100 m, c'est-à-dire un point pour 1 ha. Cette distribution régulière des points de sondage sur l'ensemble de la population procure fréquemment plus d'informations par unité de coût, comparé à un échantillonnage aléatoire (Rondeux, 1999). Selon Massenet (2010), dans un peuplement de feuillus, une placette représentative doit comporter idéalement un nombre minimum de 20 à 25 (voire 30) arbres. Ainsi, les placettes posées sont de dimensions $20 \mathrm{~m}$ x $15 \mathrm{~m}$ soit 3 ares dans les parcelles de 5 à 15 ans. Elles sont de dimensions de $30 \mathrm{~m}$ x $30 \mathrm{~m}$ soit 9 ares dans les peuplements d'âge supérieur à 15 ans. Dans chaque placette, tous les arbres ont été numérotés avec l'aérosol. Le diamètre de tous les arbres à hauteur de poitrine (Dbh) a été mesuré, les hauteurs totales de trois arbres moyens ont été aussi mesurées. La hauteur des 2 plus gros arbres a été mesurée par placette de 3 ares de superficie et des 8 plus gros arbres par placette de 9 ares dans les parcelles âgées de plus 15 ans pour éviter la sousestimation de la hauteur dominante (Pardé et Bouchon, 1988). Les hauteurs totales ont été prises avec le clinomètre «SUUNTO» à une distance déterminée de l'arbre $(d)$. La première visée ( $\alpha \%$ ) a été faite au sommet de l'arbre, donc à l'extrémité du bourgeon terminal et la seconde au pied de l'arbre $(\beta \%)$. La formule $H=d \times \frac{\alpha-\beta}{100}$ a permis d'estimer la hauteur.

\section{Traitements et analyses statistiques Traitement des données \\ Les paramètres dendrométriques suivants ont été calculés:}




\section{Densité}

La densité $(N)$ qui est le nombre de pieds à

l'hectare, est obtenue comme suit :

$$
\mathrm{N}=(10000 \times \mathrm{n}) / \mathrm{s}
$$

avec $n$ le nombre de pieds à l'intérieur de la placette échantillon de surface $s$ exprimée en $\mathrm{m}^{2}$.

\section{Diamètre quadratique moyen $(D g)$}

Le diamètre de l'arbre de surface terrière moyenne $(D g)$ a été déterminé par la formule suivante:

$$
\mathrm{D}_{\mathrm{g}}=\sqrt{\frac{1}{\mathrm{n}} \sum_{\mathrm{i}=1}^{\mathrm{n}} \mathrm{d}_{\mathrm{i}}^{2}}
$$

Avec di le diamètre de l'arbre $i$ de la placette considérée et $\mathrm{n}$ le nombre de pieds d'arbres dans la placette.

\section{$>\quad$ Hauteur moyenne $(\mathrm{Hm})$}

La hauteur moyenne $(\mathrm{Hm})$ a été obtenue à partir de la moyenne arithmétique des hauteurs de tous les arbres échantillonnés $(n)$. La relation est la suivante:

$H_{m}=\Sigma H_{i} / n$

$H i$, la hauteur de l'arbre $i$ et $n$, le nombre total d'arbres.

\section{$>\quad$ Hauteur dominante $(\mathrm{Ho})$}

La hauteur dominante $(\mathrm{Ho})$ est calculée à partir de la moyenne arithmétique des hauteurs des plus gros arbres échantillonnés par placette.

$>\quad$ Surface terrière $\left(G\right.$, en $\left.\mathrm{m}^{2} / \mathrm{ha}\right)$

La surface terrière $(G)$ est la somme des surfaces des sections transversales de tous les arbres du peuplement à hauteur de référence ( $1,30 \mathrm{~m}$ du sol) . Elle a été déterminée comme suit:

$$
\mathrm{G}=\frac{0,0001 \pi}{4 \mathrm{~s}} \sum_{\mathrm{i}=1}^{\mathrm{n}} \mathrm{d}_{\mathrm{i}}^{2} \quad \text { Avec } G=\text { la surface }
$$

terrière en $\mathrm{m}^{2} / \mathrm{ha} ; d_{\mathrm{i}}=$ diamètre en centimètre (cm) de l'arbre $i$ du placeau et $\mathrm{s}=$ surface $\mathrm{du}$ placeau en ha. Elle s'exprime en $\mathrm{m}^{2} / \mathrm{ha}$.

\section{$>\quad$ Facteur d'espacement de Hart-}

Becking $(S \%)$

C'est le ratio entre l'écartement moyen des arbres et la hauteur dominante du peuplement. En ce qui concerne le teck, c'est le paramètre qui est utilisé pour guider les trois premières éclaircies. Il s'exprime en \% et se détermine de la façon suivante:

$$
\mathbf{s} \%=100 \times\left(\frac{\mathrm{e}}{\mathrm{Ho}}\right)
$$

Où $H o$ la hauteur dominante, $e$ désigne l'écartement moyen entre les arbres et calculé par la formule:

$$
\mathrm{e}=\sqrt{\frac{1000 \mathrm{C}}{\mathrm{N}}}
$$

$N$ étant le nombre d'arbres à l'hectare lorsque les arbres occupent les sommets de carrés.

\section{Analyse statistique \\ Paramètres dendrométriques}

Les analyses statistiques constituées de tests de comparaison de moyennes ou de médianes des paramètres dendrométriques ont été effectuées pour un âge de plantation fixé. Plus spécifiquement, pour un âge de plantation donné $(5,10,15,25$ ou 35 ans), la normalité de la distribution de chaque paramètre dendrométrique est vérifiée par un test de Shapiro-Wilk et l'homogénéité des variances suivant les trois types de sol (vertisol, ferrugineux, ferralitique) à l'aide d'un test de Levene. Au besoin, des transformations de variables sont faites pour remplir les deux conditions susmentionnées. Lorsque ces conditions sont respectées, une analyse de variance est effectuée pour vérifier l'existence d'une différence significative entre les moyennes du paramètre suivant le type de sol. La comparaison des moyennes a été faite à l'aide du test de Student-Newman-Keuls. Lorsque ces conditions ne sont pas vérifiées même après la transformation des variables, le test non-paramétrique de Kruskal-Wallis est utilisé. En cas de différence significative, le test de Wilcoxon-Mann-Whitney de comparaisons multiples par paires avec une correction de Bonferroni est effectué pour l'identification des différences entre les médianes pour le paramètre donné (Hochberg, 1988).

Structure en diamètre

Tous les individus ont été répartis dans des classes de diamètre d'amplitude $5 \mathrm{~cm}$. Ainsi, les densités d'arbres (en arbres/ha) par classes de diamètre ont été déterminées. Des histogrammes ont été construits par âge et par type de sol pour caractériser la répartition des individus dans les classes de diamètres. 


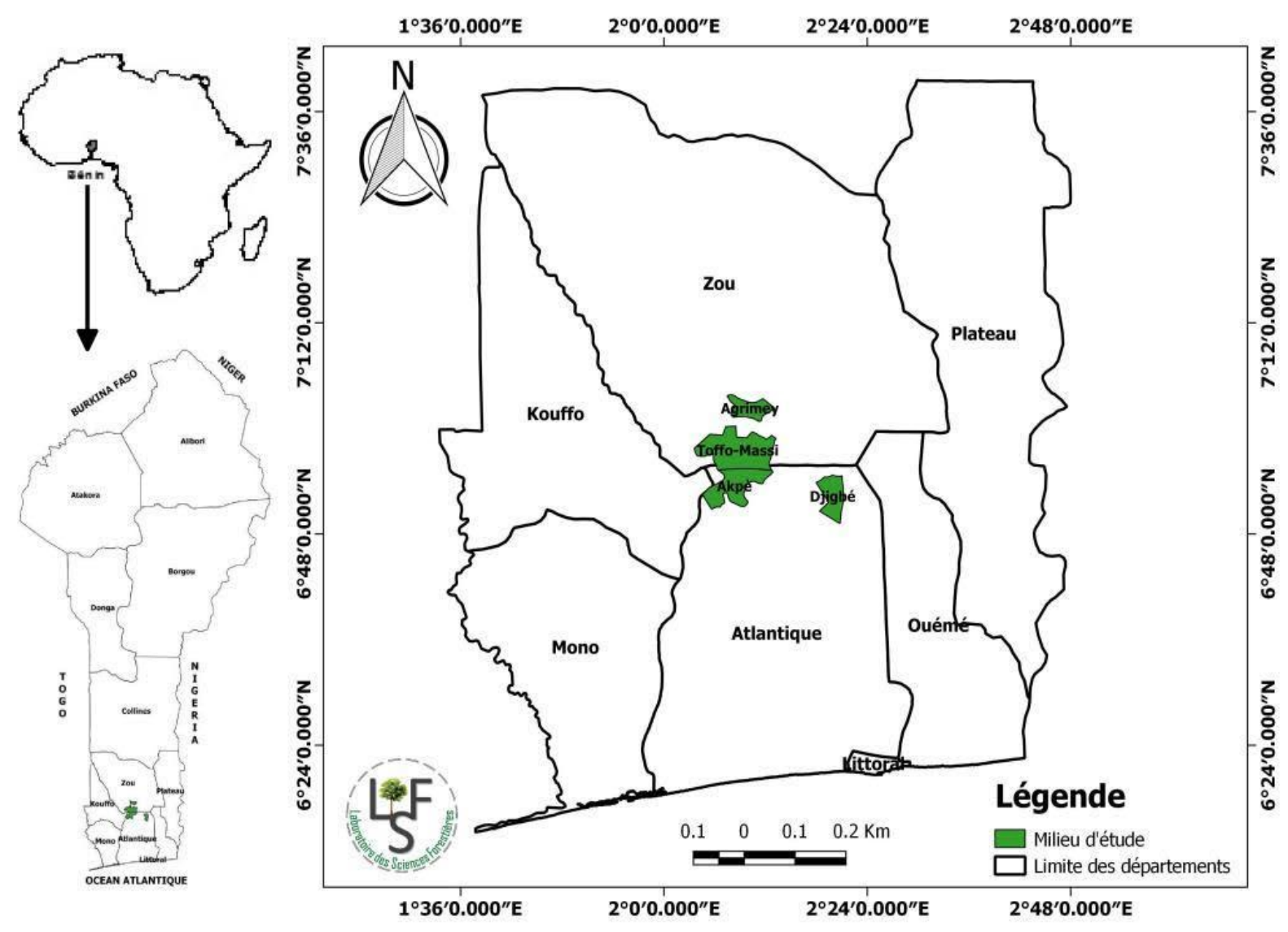

Figure 1: Localisation des zones d'études des caractéristiques structurales (LSF/UAC).

\section{RÉSULTATS}

Caractéristiques structurales des peuplements âgés de 5 ans en fonction des types de sol

\section{Paramètres dendrométriques}

Dans les peuplements de 5 ans, les densités moyennes sont statistiquement égales, d'un type de sol à l'autre $(\mathrm{P}=0,297)$. De même, la surface terrière $(\mathrm{P}=0,6)$ et le facteur d'espacement $(\mathrm{P}=0,655)$ ne varient pas significativement suivant les types de sols. Par contre, le diamètre moyen $(\mathrm{P}=0,000)$, la hauteur moyenne $(\mathrm{P}=0,000)$ et la hauteur dominante $(\mathrm{P}=0,000)$ sont significativement différentes suivant les types de sols. Le diamètre moyen des pieds de teck installés sur le sol ferrugineux $(6,03 \mathrm{~cm} \pm 0,97)$ est significativement plus faible que ceux des pieds de teck installés sur le vertisol $(7,79 \mathrm{~cm}$ $\pm 1,64)$ et sur sol ferralitique $(8,24 \mathrm{~cm} \pm 1,68)$. Le sol ferralitique est celui sur lequel les hauteurs moyenne $(8,52 \mathrm{~m} \mathrm{~cm} \pm 1,39)$ et dominante $(9,53 \mathrm{~m} \pm 1,81)$ sont les plus élevées à 5 ans d'âge (Tableau 1).

\section{Structure en diamètre}

Les peuplements des trois types de sols présentent globalement des distributions des individus jeunes ou de petits diamètres (Figure 2). Les jeunes individus de teck $(5 \mathrm{~cm}$ et $10 \mathrm{~cm}$ de diamètre) sont les plus représentés à l'hectare suivis des individus de diamètre allant de $0 \mathrm{~cm}$ à $5 \mathrm{~cm}$ pour le peuplement évoluant sur sol ferrugineux et les individus de diamètre compris entre $10 \mathrm{~cm}$ et $15 \mathrm{~cm}$ 
pour les peuplements évoluant sur sol ferralitique et vertisol. Il y a une quasiabsence d'individus de diamètre compris entre 10 et $15 \mathrm{~cm}$ dans le peuplement sur sol ferrugineux.

\section{Caractéristiques structurales des peuplements âgés de 10 ans en fonction des types de sol \\ Paramètres dendrométriques}

Dans les peuplements de 10 ans, à part les densités qui ne diffèrent pas significativement $(\mathrm{P}=0,183)$, le type de sol a une influence hautement significative sur le diamètre moyen, les hauteurs moyenne et dominante, la surface terrière et le facteur d'espacement $(p=0,000)$. Les plus fortes valeurs moyennes du diamètre $(16,53 \mathrm{~cm} \pm$ $2,75)$, de la hauteur moyenne (14,76 $\mathrm{m} \pm$ $1,44)$ de la hauteur dominante $(16,73 \mathrm{~m} \pm$ $1,85)$ et de la surface terrière $(15,03 \mathrm{~m} \pm$ $6,98)$ ont été obtenues dans les peuplements installés sur du vertisol. Contrairement aux autres paramètres dendrométriques, le facteur d'espacement a donné la plus faible valeur $(23,81 \pm 6,59)$ sur le vertisol et la plus forte $(41,22 \pm 7,07)$ a été remarquée sur le sol ferrugineux (annexe).

\section{Structure en diamètre}

Tous les peuplements évoluant sur les sols ferralitiques et vertisol présentent globalement des distributions des individus jeunes ou de petits diamètres (Figure 3). Par ailleurs, le peuplement du sol ferrugineux présente une distribution d'individus de petits diamètres. La classe modale des peuplements varie d'un sol à l'autre. Les individus de diamètre compris entre $5 \mathrm{~cm}$ et $10 \mathrm{~cm}$ sont les plus représentés pour le peuplement sur sol ferrugineux. Pour le peuplement sur sol ferralitique, les individus les plus représentés sont ceux de la classe 10 à $15 \mathrm{~cm}$. Pour le peuplement sur vertisol, il s'agit de la classe de 15 à $20 \mathrm{~cm}$. Les individus de diamètre supérieur à $15 \mathrm{~cm}$ sont fortement représentés à l'hectare dans le peuplement sur vertisol, moyennement dans le peuplement sur sol ferralitique et très faiblement sur sol ferrugineux.

\section{Caractéristiques structurales des} peuplements âgés de 15 ans en fonction des types de sol

\section{Paramètres dendrométriques}

L'analyse des peuplements de 15 ans montre que le type de sol influence significativement le facteur d'espacement $(\mathrm{P}=0,033)$. Du point de vue diamètre moyen, hauteur moyenne, hauteur dominante et surface terrière et suivant les différents types de sols, la différence est hautement significative $(\mathrm{P}=0,000)$. Les plus fortes valeurs moyennes de diamètre $(22,79 \pm 2,97)$, hauteur moyenne $(17,6 \pm 0,97)$, hauteur dominante $(18,68 \pm 1,36)$ et surface terrière $(11,16 \pm 2,11)$ ont été remarquées sur sol ferralitique. Quant à la densité, elle est forte sur sol ferrugineux $(571,93 \pm 174,36)$ et plus faible sur le vertisol $(276,67 \pm 51,97)$ (Tableau 1).

\section{Structure en diamètre}

Tous les peuplements évoluant sur les sols ferralitiques et vertisol présentent globalement des distributions des individus jeunes ou de petits diamètres (Figure 4). Par ailleurs, le peuplement du sol ferrugineux présente une allure décroissante avec une prédominance relative d'individus de petits diamètres. La classe modale varie d'un peuplement à l'autre. Les individus de diamètre compris entre $20 \mathrm{~cm}$ et $25 \mathrm{~cm}$ sont les plus représentés pour les peuplements installés sur sol ferralitique et vertisol. La classe modale est 5 à $10 \mathrm{~cm}$ pour le peuplement sur sol ferrugineux.. Sur ce sol, les arbres de faible diamètre $(<15 \mathrm{~cm})$ sont bien représentés à l'hectare alors que leur densité est faible sur les deux autres types de sol. Sur sol ferralitique ou vertisol, les arbres de teck de classe de diamètre 20 à $25 \mathrm{~cm}$ sont les plus représentés à l'hectare. Cette classe de diamètre est pratiquement absente sur le sol ferrugineux. 
Caractéristiques structurales des peuplements âgés de $\mathbf{2 5}$ ans en fonction des types de sol

\section{Paramètres dendrométriques}

Dans les peuplements de 25 ans, il ressort que du point de vue diamètre moyen, hauteur moyenne et dominante, il y a une différence hautement significative suivant les types de sol $(\mathrm{P}=0,000)$. C'est sur le sol ferrugineux que la plus forte densité moyenne $(282,05 \pm 70,79)$ a été observée. Les plus grandes valeurs de diamètre $(29,3 \pm 3,24)$ de hauteur moyenne $(14,9 \pm 1,2)$ et dominante $(15,67 \pm 1,15)$ ont été remarquées sur le vertisol avec la plus faible densité $(113,95 \pm$ 37,04). Par contre, il n'y a pas de différence significative quant à la surface terrière $(\mathrm{P}=$ $0,498)$ et le facteur d'espacement $(\mathrm{P}=0,647)$ suivant les types de sol (Tableau 1).

\section{Structure en diamètre}

Tous les peuplements évoluant sur sol ferralitique et ferrugineux présentent globalement des distributions des individus jeunes ou de petits diamètres (Figure 5). Le sol ferralitique de cette catégorie d'âge abrite des arbres de diamètre compris entre 40 et 45 $\mathrm{cm}$ et entre 45 et $50 \mathrm{~cm}$. Les individus du teck de cette grosseur sont quasi absents sur le sol ferrugineux. Par contre, le peuplement sur vertisol présente une distribution caractéristique d'une prédominance d'individus de gros diamètres.

\section{Caractéristiques structurales des peuplements âgés de $\mathbf{3 5}$ ans en fonction des types de sol \\ Paramètres dendrométriques}

De l'analyse des peuplements de 35 ans, il ressort que le type de sol n'a pas d'influence significative sur le diamètre, la hauteur moyenne, la hauteur dominante et la surface terrière $(\mathrm{P}>0,05)$. La plus forte densité est observée sur le vertisol suivi du sol ferralitique (Tableau 1).

\section{Structure en diamètre}

Tous les peuplements des trois types de sol présentent globalement une distribution de prédominance d'individus jeunes ou de petits diamètres (Figure 6). La classe modale est de 25 à $30 \mathrm{~cm}$ sur le sol ferralitique et le vertisol et de 20 à 25 sur sol ferrugineux. On note sur les trois types de sols (ferralitiques, ferrugineux et vertisol) de cette catégorie d'âge, la présence des arbres de gros diamètre allant de $35 \mathrm{~cm}$ à $45 \mathrm{~cm}$.

Tableau 1: Paramètres dendrométriques des peuplements en fonction de l'âge et des types de sol.

\begin{tabular}{|c|c|c|c|c|c|c|c|}
\hline Age & Sol & Densité & $\begin{array}{l}\text { Diamètre } \\
\text { moyen } \\
\text { (cm) }\end{array}$ & $\begin{array}{l}\text { Hauteur } \\
\text { moyenne } \\
\text { (m) }\end{array}$ & $\begin{array}{c}\text { Hauteur } \\
\text { dominante } \\
\text { (m) }\end{array}$ & $\begin{array}{c}\text { Surface } \\
\text { Terrière } \\
\left(\mathbf{m}^{2}\right)\end{array}$ & $\begin{array}{c}\text { Facteur } \\
\text { d'espacement } \\
(\%)\end{array}$ \\
\hline \multirow{6}{*}{5} & Ferralitique & 941,67 & 8,24 & 8,52 & 9,53 & 5,21 & 48,43 \\
\hline & & $\pm 640,73$ (a) & $\pm 1,68$ (a) & $\pm 1,39$ (a) & $\pm 1,81(\mathrm{a})$ & $\pm 3,63$ (a) & $\pm 36,79$ (a) \\
\hline & Ferrugineux & 1106,94 & 6,03 & 5,8 & 7,01 & 3,23 & 49,51 \\
\hline & & $\pm 415,52(\mathrm{a})$ & $\pm 0,97$ (b) & $\pm 0,88$ (c) & $\pm 1,17$ (b) & $\pm 1,48$ (a) & $\pm 23,66$ (a) \\
\hline & Vertisol & 837,5 & 7,79 & 7,15 & 8,15 & 4,5 & 50,95 \\
\hline & & $\pm 445,59$ (a) & $\pm 1,64$ (a) & $\pm 1,61(\mathrm{~b})$ & $\pm 1,38(\mathrm{c})$ & $\pm 3,93$ (a) & $\pm 23,73$ (a) \\
\hline \multicolumn{2}{|c|}{ Probabilité } & $\mathrm{P}=0,297$ & $\mathrm{P}=0,00002$ & $\mathrm{P}=0,00000$ & $\mathrm{P}=0,00006$ & $\mathrm{P}=0,6$ & $\mathrm{P}=0,655$ \\
\hline \multirow{6}{*}{10} & Ferralitique & 608,89 & 14,59 & 11,3 & 11,89 & 10,17 & 36,75 \\
\hline & & $\pm 227,29$ (a) & $\pm 3,31$ (c) & $\pm 0,96$ (b) & $\pm 0,9$ (b) & $\pm 5,06(\mathrm{~b})$ & $\pm 9,77$ (b) \\
\hline & Ferrugineux & 695,37 & 10,51 & 8,27 & 9,48 & 6,08 & 41,22 \\
\hline & & $\pm 109,3$ (a) & $\pm 1,5(\mathrm{~b})$ & $\pm 1,44(\mathrm{c})$ & $\pm 1,37$ (c) & $\pm 1,74$ (c) & $\pm 7,07$ (a) \\
\hline & Vertisol & 701,96 & 16,53 & 14,76 & 16,73 & 15,03 & 23,81 \\
\hline & & $\pm 164,71$ (a) & $\pm 2,75$ (a) & $\pm 1,44$ (a) & $\pm 1,85$ (a) & $\pm 6,98$ (a) & $\pm 6,59(\mathrm{c})$ \\
\hline
\end{tabular}


D. GNANGUENON-GUESSE et al. / Int. J. Biol. Chem. Sci. 11(5): 2119-2132, 2017

\begin{tabular}{|c|c|c|c|c|c|c|c|}
\hline \multicolumn{2}{|c|}{ Probabilité } & $\mathrm{P}=0,1836$ & $\mathrm{P}=0,00000$ & $\mathrm{P}=0,00000$ & $\mathrm{P}=0,00000$ & $\mathrm{P}=0,00000$ & $\mathrm{P}=0,00000$ \\
\hline \multirow{6}{*}{15} & Ferralitique & 286,11 & 22,79 & 17,6 & 18,68 & 11,16 & 33,07 \\
\hline & & $\pm 86,11(\mathrm{~b})$ & $\pm 2,97$ (a) & $\pm 0,97$ (a) & $\pm 1,36$ (a) & $\pm 2,11$ (a) & $\pm 6,51$ (a) \\
\hline & Ferrugineux & 571,93 & 11,09 & 10,33 & 11,05 & 5,41 & 40,64 \\
\hline & & $\pm 174,36(\mathrm{a})$ & $\pm 1,94(\mathrm{~b})$ & $\pm 1,43(\mathrm{c})$ & $\pm 1,37(\mathrm{c})$ & $\pm 1,86(\mathrm{~b})$ & $\pm 12,23$ (a) \\
\hline & Vertisol & 276,67 & 21,13 & 15,12 & 16,15 & 9,73 & 38,07 \\
\hline & & $\pm 51,97(\mathrm{~b})$ & $\pm 1,77$ (a) & $\pm 1,2(\mathrm{~b})$ & $\pm 1,41(b)$ & $\pm 2,37$ (a) & $\pm 5,78(\mathrm{a})$ \\
\hline \multicolumn{2}{|c|}{ Probabilité } & $\mathrm{P}=0,00000$ & $\mathrm{P}=0,00000$ & $\mathrm{P}=0,00000$ & $\mathrm{P}=0,00000$ & $\mathrm{P}=0,00000$ & $\mathrm{P}=0,0335$ \\
\hline \multirow{6}{*}{25} & Ferralitique & 219,44 & 29,3 & 18,55 & 19,48 & 13,6 & 41,35 \\
\hline & & $\pm 140,95$ (a) & $\pm 3,24$ (b) & $\pm 1,48(\mathrm{~b})$ & $\pm 2,17$ (b) & $\pm 7,11$ (a) & $\pm 19,16$ (a) \\
\hline & Ferrugineux & 282,05 & 21,89 & 14,9 & 15,67 & 10,28 & 38,75 \\
\hline & & $\pm 70,79$ (a) & $\pm 2,16(\mathrm{c})$ & $\pm 1,2(\mathrm{c})$ & $\pm 1,15$ (c) & $\pm 0,92$ (a) & $\pm 3,57$ (a) \\
\hline & Vertisol & 113,95 & 39,12 & 23,47 & 23,71 & 14,05 & 43,87 \\
\hline & & $\pm 37,04(\mathrm{~b})$ & $\pm 3,74$ (a) & $\pm 2,42(\mathrm{a})$ & $\pm 2,43$ (a) & $\pm 5,14$ (a) & $\pm 19,19$ (a) \\
\hline \multicolumn{2}{|c|}{ Probabilité } & $\mathrm{P}=0,00000$ & $\mathrm{P}=0,00000$ & $\mathrm{P}=0,00000$ & $\mathrm{P}=0,00000$ & $\mathrm{P}=0,498$ & $\mathrm{P}=0,647$ \\
\hline \multirow{6}{*}{35} & Ferralitique & 225,4 & 27,94 & 19,74 & 20,45 & 13,24 & 33,67 \\
\hline & & $\pm 70,87$ (a) & $\pm 5,29$ (a) & $\pm 2,3$ (a) & $\pm 2,51(\mathrm{a})$ & $\pm 3,04$ (a) & $\pm 4,22$ (b) \\
\hline & Ferrugineux & 181,89 & 31,33 & 19,99 & 20,48 & 11,39 & 41,74 \\
\hline & & $\pm 117,23(b)$ & $\pm 7,9$ (a) & $\pm 3,39$ (a) & $\pm 2,92$ (a) & $\pm 3,14$ (a) & $\pm 12,64$ (b) \\
\hline & Vertisol & 230,37 & 30,59 & 19,73 & $20,31 \pm$ & 16,83 & 33,02 \\
\hline & & $\pm 43,33$ (a) & $\pm 1,57$ (a) & $\pm 1,31(\mathrm{a})$ & 1,46 (a) & $\pm 2,83$ (a) & $\pm 4,11(\mathrm{~b})$ \\
\hline \multicolumn{2}{|c|}{ Probabilité } & $\mathrm{P}=0,03077$ & $\mathrm{P}=0,8357$ & $\mathrm{P}=0,8761$ & $\mathrm{P}=0,8652$ & $\mathrm{P}=0,0913$ & $\mathrm{P}=0,00306$ \\
\hline
\end{tabular}

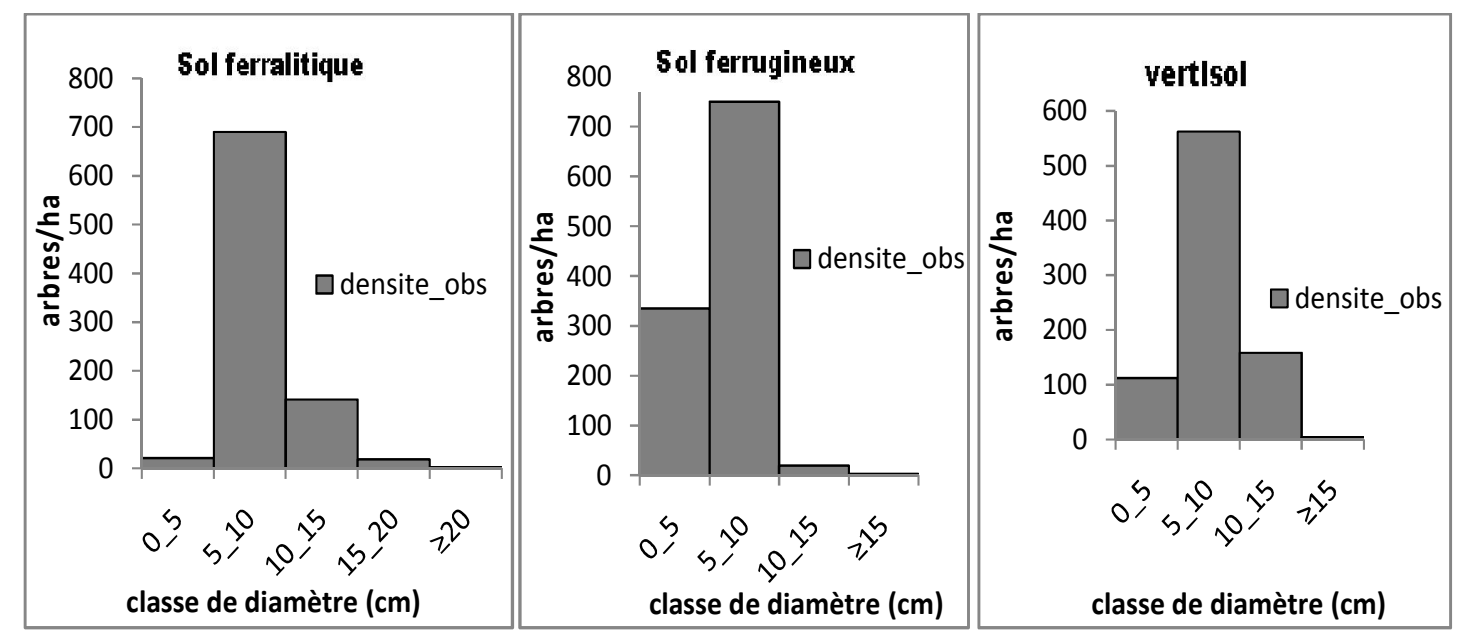

Figure 2: Structure en diamètre des peuplements âgés de 5 ans en fonction des types de sol. 

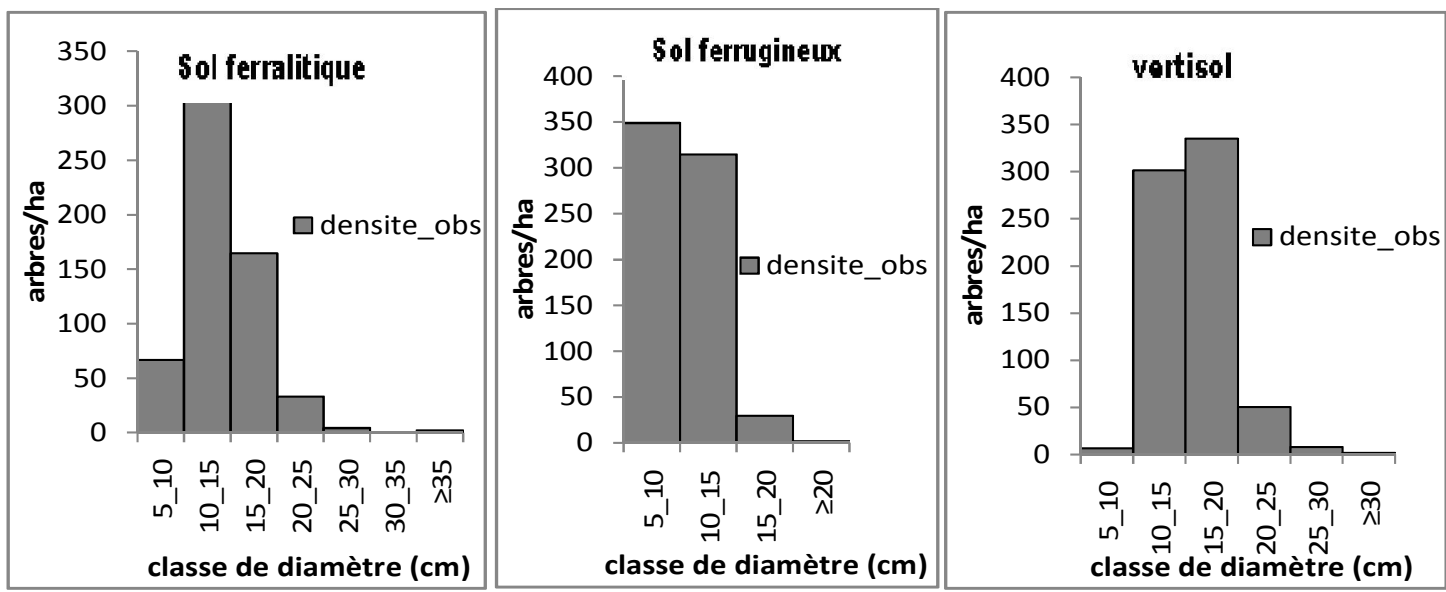

Figure 3: Structure en diamètre des peuplements âgés de 10 ans en fonction des types de sol.
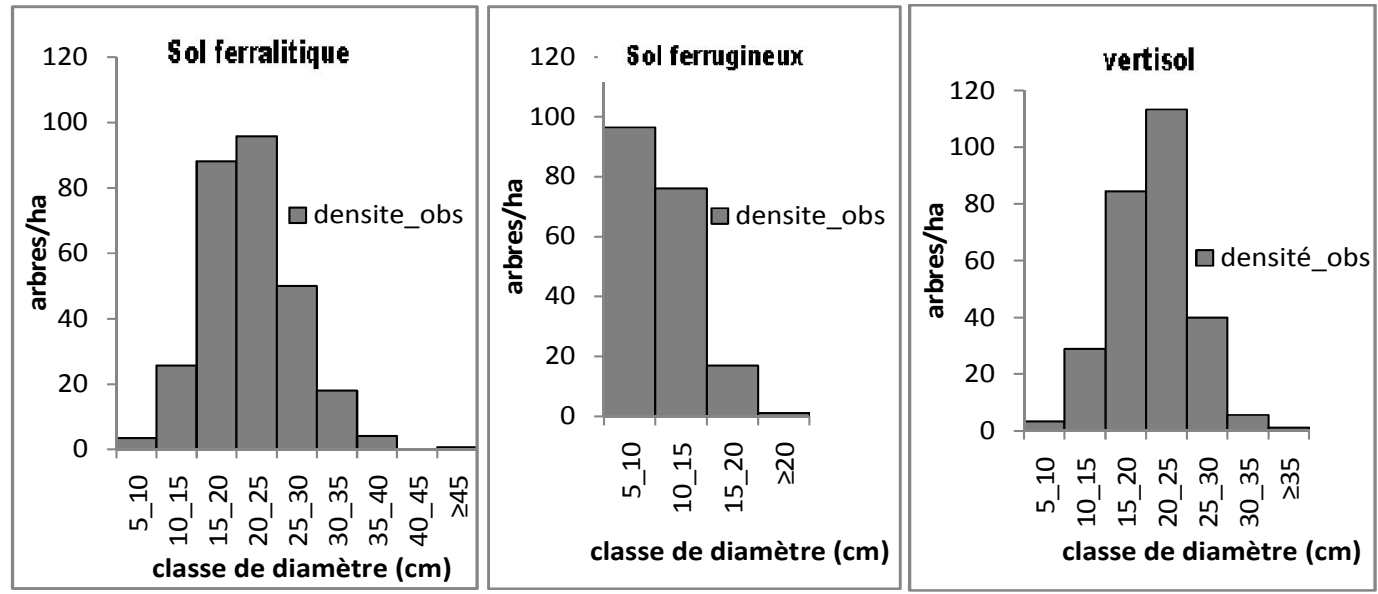

Figure 4: Structure en diamètre des peuplements âgés de 15 ans en fonction des types de sol.

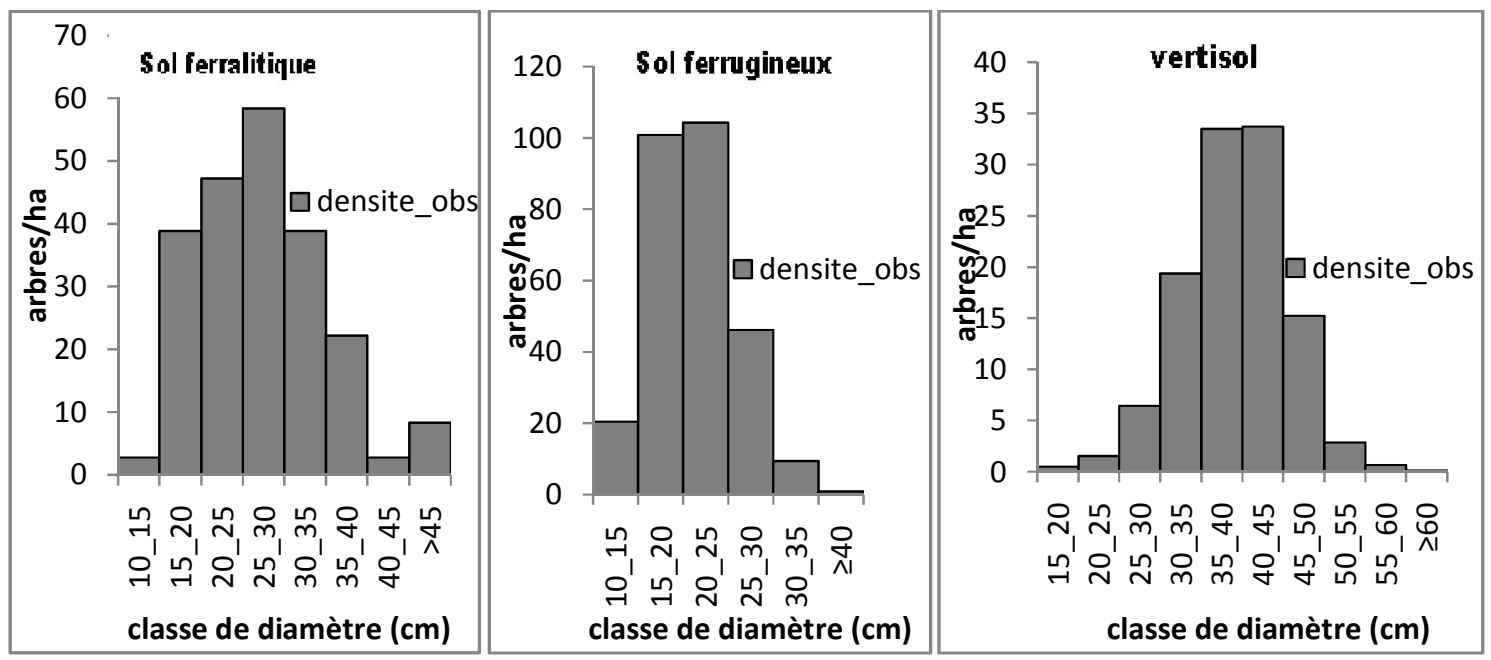

Figure 5: Structure en diamètre des peuplements âgés de 25 ans en fonction des types de sol. 


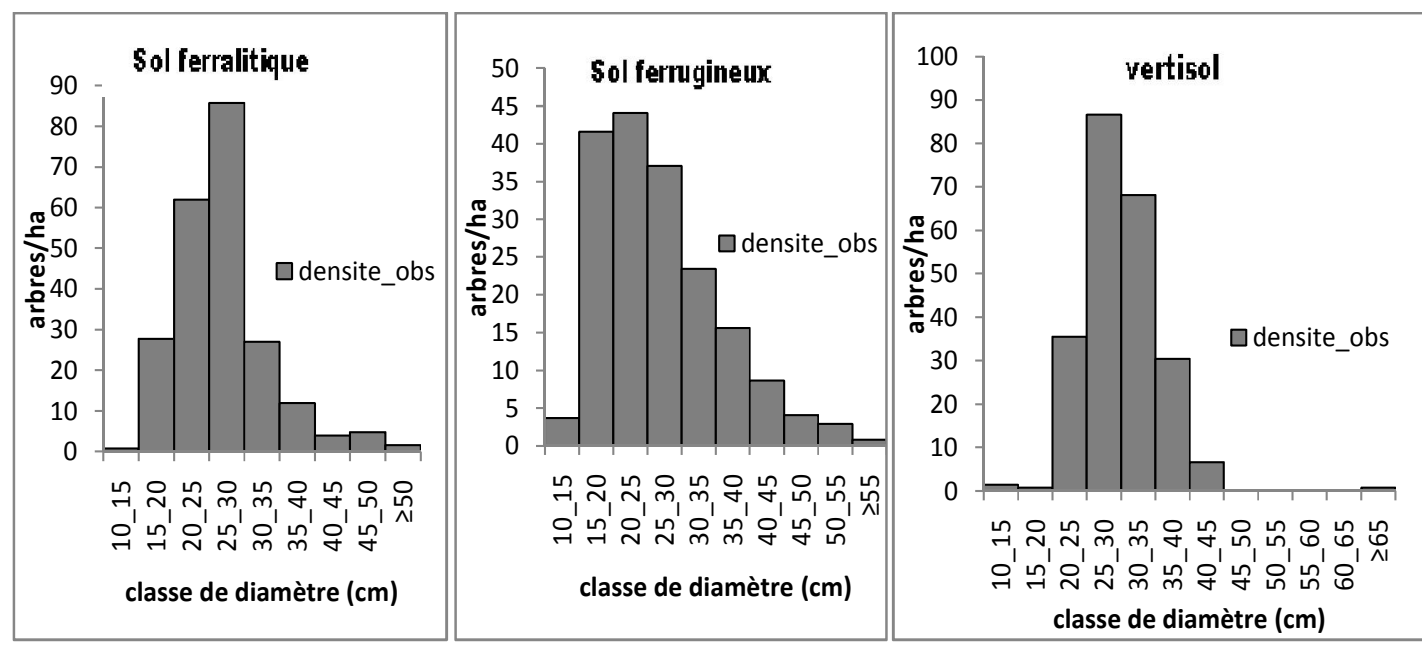

Figure 6: Structure en diamètre des peuplements âgés de 35 ans en fonction des types de sol.

\section{DISCUSSION}

Caractéristiques dendrométriques des
plantations
Densité

Les paramètres dendrométriques en termes de densité, diamètre de l'arbre de la surface terrière moyenne, la surface terrière, la hauteur moyenne, la hauteur dominante et le facteur d'espacement sont des données chiffrées pour affiner la description et mieux comprendre l'évolution d'une parcelle (Massenet, 2010). Les densités observées pour les catégories d'âges (5 et 10 ans) sont significativement égales d'un type de sol à un autre. En effet, dans les différents peuplements de 5 ans échantillonnés, les nombres d'arbres à l'hectare varient de 837 à $1107 \mathrm{arbres} / \mathrm{ha}$. Ce qui est conforme aux résultats de Kpadonou (2015) qui a trouvé une densité de 1062 arbres/ha dans le secteur forestier d'Agrimey. Ils confirment les observations de Akouèhou et Ayélo (2005) dans les peuplements des régénérations naturelles de Djigbé. Toutefois, ces résultats ne correspondent pas aux prescriptions de Trainer et al. (2003) qui recommandent d'adopter dans les plantations de teck une densité initiale de 1500 arbres/ha. Ces faibles densités (837 à 1107 arbres/ha) obtenues dans ces peuplements de 5 ans provenant des régénérations naturelles sont dues aux retards observés ces dernières années dans la mise en œuvre des coupes de régénérations naturelles. Les dégagements des bandes ne sont plus réalisés avant les pluies. Ce qui entraîne l'échec desdites régénérations naturelles.

Les densités des peuplements de 10 ans d'âge (609; 695 et $701 \quad$ plants/ha) respectivement sur les sols ferralitiques, ferrugineux et vertisols sont obtenues après la première éclaircie. Elles ne sont pas trop conformes aux prescriptions de Trainer et al. (2003) au Bénin qui recommandent que la première éclaircie réduise la densité du peuplement à 750 plants à l'hectare. L'absence de rigueur et du suivi lors des prélèvements en éclaircie serait à la base de ce constat. Les densités obtenues dans les peuplements des trois types de sols présentent une différence hautement significative suivant les catégories d'âges: 15 ans, 25 ans et 35 ans. Cette différence observée serait due aux types de sols qui n'ont pas permis un bon développement des arbres comme le sol ferrugineux, aux densités initiales des peuplements et à l'absence de rigueur et de suivi dans la mise en œuvre des différentes éclaircies. 
Diamètre moyen, hauteur moyenne et hauteur dominante

Les résultats de cette étude montrent qu'à l'exception du diamètre moyen, la hauteur moyenne et la hauteur dominante des peuplements de 35 ans, tous ces paramètres dendrométriques des peuplements de teck varient suivant les types de sol et par catégorie d'âge. Le type de sol a influencé significativement les paramètres dendrométriques comme le diamètre moyen, la hauteur moyenne et la hauteur dominante. Aucune différence significative n'existe au niveau $\mathrm{du}$ diamètre moyen, la hauteur moyenne et la hauteur dominante des peuplements de 35 ans installés sur des sols ferralitiques, ferrugineux et vertisol. Le type de sol a une influence significative sur le diamètre moyen dans les peuplements de 5 ans 10 ans, 15 ans et 25 ans). Les peuplements installés sur le sol ferralitique et le vertisol présentent les fortes valeurs de diamètre moyen du teck $(8,24 \mathrm{~cm} ; 7,79 \mathrm{~cm})$ pour les peuplements de 5 ans, $(14,59 \mathrm{~cm} ; 16,53$ $\mathrm{cm})$ pour les peuplements de 10 ans $(22,79$ $\mathrm{cm} ; 21,13 \mathrm{~cm}$ ) pour les peuplements de 15 ans et $(29,3 \mathrm{~cm} ; 39,12 \mathrm{~cm})$ pour les peuplements de 25 ans. Ces caractéristiques sont comparables à celles rapportées par Toundoh (2013) pour les mêmes plantations âgées de 18 à 24 ans qui ont un diamètre quadratique moyen de $30 \mathrm{~cm}$ à $38 \mathrm{~cm}$. Ces résultats se rapprochent également de ceux de Trainer et al. (2003) (34 à $38 \mathrm{~cm}$ ) obtenus dans les plantations de teck âgées de 19 à 26 ans en Côte d'Ivoire. Les faibles diamètres moyens obtenus ont été globalement remarqués sur les sols ferrugineux. Ce sol aurait un degré de fertilité moindre en comparaison avec les autres types de sol. Il est à noter que les diamètres moyens $(29,3 \mathrm{~cm}$ et $39,12 \mathrm{~cm})$ des arbres des peuplements âgés de 25 ans sont respectivement supérieurs aux diamètres moyens $(27,94 \mathrm{~cm}$ et $30,59 \mathrm{~cm})$ des arbres de peuplements de 35 ans sur des sols ferralitiques et vertisol. Cette contreperformance observée au niveau des peuplements de 35 ans sur ces sols serait due à la forte densité du sous-bois avec des rejets de souches et à la concurrence des sujets du teck avec les lianes épiphytes constatés sur lesdits sols. Par contre, le diamètre moyen $(31,33$ $\mathrm{cm})$ des arbres du peuplement de 35 ans est supérieur à celui $(21,89 \mathrm{~cm})$ des arbres du peuplement de 25 ans du sol ferrugineux. Tous ces résultats confirment la thèse de Herrero-Jáuregui et al. (2012) selon laquelle la distribution par classes de diamètre varie en fonction des facteurs du milieu tels que les conditions environnementales, les conditions climatiques, les processus de régénération, la concurrence pour les ressources et est fonction de l'âge et du type de sol.

\section{Surface terrière}

La connaissance de la surface terrière permet de suivre l'évolution du peuplement dans son ensemble, pour quantifier l'intensité de l'éclaircie. Elle permet la comparaison rapide de différents peuplements. Le ralentissement de sa croissance traduit une saturation de la capacité de production de la plantation et induit l'intervention d'une éclaircie dans les peuplements âgés. Selon les prescriptions de Trainer et al. (2003), elle est utilisée pour évaluer l'intervention des éclaircies dans une plantation. Sa norme selon les mêmes auteurs varie entre $15 \mathrm{~m}^{2} /$ ha et 21 $\mathrm{m}^{2} /$ ha. Au-delà de la limite supérieure, il faudrait procéder à une éclaircie. Les résultats de cette étude révèlent que la surface terrière ne présente aucune différence significative suivant les types de sols (ferralitique, sol ferrugineux et vertisol) dans les peuplements âgés de 25 ans et 35 ans.

\section{Facteur d'espacement}

Le facteur d'espacement comme la surface terrière permet de suivre la dynamique $\mathrm{du}$ peuplement dans son ensemble, pour identifier les moments propices des éclaircies et quantifier leurs intensités dans les jeunes peuplements. Il est un des meilleurs indicateurs de la structure du peuplement puisqu'il le décrit en trois dimensions par le truchement de la hauteur dominante mise en parallèle avec la distance moyenne au sol des arbres entre eux (Sinsin, 2007 ; Blanchin et al. 2009). Selon les prescriptions de Trainer et al. (2003), elle est utilisée pour évaluer 
l'intervention des éclaircies dans une jeune plantation. Sa norme selon les mêmes auteurs varie entre $22 \%$ et $33 \%$. En dessous de cette norme, il faut éclaircir. Les résultats de cette étude révèlent qu'à l'exception des peuplements de 5 ans, le facteur d'espacement présente une différence significative suivant les types de sols (ferralitique, sol ferrugineux et vertisol) dans les jeunes peuplements de 10 ans et 15 ans.

\section{Structure en diamètre des peuplements de teck en fonction du sol et de l'âge}

L'analyse des peuplements évoluant sur du sol ferrugineux présentent globalement une distribution des individus jeunes ou de petits diamètres. Les peuplements âgés de 10 et 15 ans présentent une allure décroissante avec une prédominance relative d'individus de petits diamètres et une réduction progressive $\mathrm{du}$ nombre d'arbres dans les classes supérieures de diamètre. Le faible développement des plants observés dans ces peuplements serait dû non seulement à l'appauvrissement du sol ferrugineux mais aussi aux multiples feux de brousses observés ces dernières années dans les différents secteurs. De même, les peuplements du sol ferralitique de toutes les catégories d'âge présentent globalement une distribution à prédominance d'individus jeunes ou de petits diamètres. La structure des peuplements sur sol ferralitique présente un développement continu des individus au cours des années. Sur les vertisols, les peuplements d'âges inférieurs à 15 ans et ceux de 35 ans présentent globalement des individus jeunes ou de petits diamètres. Pour le teck, le vertisol a donc une très bonne capacité productive. De ces résultats, il ressort que l'hypothèse 1 selon laquelle les caractéristiques structurales des plantations de teck du Sud et du Centre Bénin varient en fonction du type de sol est vérifiée. L'hypothèse 2 «les caractéristiques structurales des plantations de teck du Sud et $\mathrm{du}$ Centre Bénin sont identiques quel que soit l'âge » est rejetée. La troisième hypothèse qui stipule que le type de sol et l'âge influencent simultanément les caractéristiques structurales des plantations de teck du sud et du centre Bénin est confirmée. Tous ces résultats concordent avec les travaux de HerreroJáuregui et al. (2012), selon qui, la distribution par classes de diamètre varie en fonction des facteurs du milieu tels que les conditions environnementales, les conditions climatiques, les processus de régénération, la concurrence pour les ressources, et est fonction de l'âge et du type de sol.

\section{Conclusion}

Les résultats des paramètres dendrométriques et la répartition des classes modales des structures des peuplements inventoriés montrent que les structures des peuplements des sols ferralitiques et vertisol présentent un développement continu des individus des teckeraies au cours des années. Le vertisol présente une capacité de production plus élevée que les sols ferralitiques et ferrugineux. Le sol ferrugineux constitue le sol le plus appauvri, avec une classe modale de 5 à $10 \mathrm{~cm}$ pour les peuplements de 5 ans, de 10 ans et de 15 ans. Même à l'âge de 35 ans, les individus les plus représentés sont dans la classe de 20 à $25 \mathrm{~cm}$. Le diamètre moyen, la hauteur moyenne et la hauteur dominante des peuplements varient significativement suivant les différents types de sol et par catégorie d'âge allant de 5 ans à 25 ans. L'accroissement de ces principaux paramètres dendrométriques entrant dans l'estimation $\mathrm{du}$ volume $\mathrm{du}$ bois se réduit considérablement à partir de 35 ans.

Il est nécessaire de proposer des directives d'aménagement forestier efficient sur la base des résultats obtenus. Sur les sols ferrugineux, la croissance des plants est très lente en diamètre et en hauteur. Il y a également une grande difficulté de recrutement dans les classes de grands diamètres.

Sur les sols ferralitiques, les peuplements de teck ont un développement acceptable. La forte densité du sous-bois avec des rejets de souches, la concurrence des sujets du teck avec les lianes épiphytes, les cas de chablis observés et la mort de certains 
individus empêchent le bon développement des plants. Il est donc souhaitable de programmer périodiquement le nettoyage de ces peuplements par les activités de désherbage et d'abattage des arbustes, afin de libérer les arbres d'élite de la concurrence.

Le teck se développe bien sur le vertisol. Pour profiter pleinement des potentialités productives de ce sol, les interventions sylvicoles notamment les travaux d'entretien sont nécessaires. D'abord, il faut protéger les plantations contre les feux de brousse et désherber régulièrement le peuplement. Dans les jeunes peuplements, il faut respecter le planning des opérations de désherbages, le balivage, les éclaircies et les autres activités sylvicoles. Dans les peuplements âgés, les dégâts de chablis font partie des problèmes sylvicoles essentiels rencontrés. Ils sont l'une des conséquences des attaques de pourridiés présents également dans ces vieux peuplements. Il faut revenir sur les bonnes pratiques de renouvellement de ces peuplements. Elles consistent à respecter la période des coupes de régénération naturelle.

\section{CONFLIT D'INTERETS}

Les auteurs déclarent qu'il n'y a aucun conflit d'intérêts.

\section{CONTRIBUTIONS DES AUTEURS}

Tous les auteurs ont contribué à la réalisation de ce travail et à la rédaction du manuscrit.

\section{REFERENCES}

Akouehou GS, Ayelo HG. 2005. De la conduite des peuplements issus de la régénération naturelle à la sylviculture: cas du teck (Tectona grandis) dans la plantation domaniale de Djigbé, commune de Zè au sud du Bénin. Bulletin de la recherche Agronomique $d u$ Bénin, 47: 7-18.

Anoumou NCA. 2007. Contribution à la gestion durable des plantations privées de teck (Tectona grandis L. f.) dans la commune de zè (département de l'atlantique) au Bénin: caractéristiques structurales, écologie et modes de gestion. Thèse d'Ingénieur Agronome, Faculté des Sciences Agronomiques, Université d'Abomey-Calavi. $142 \mathrm{p}+$ annexes.

Azankpan DJ. 2002. Etude comparative des performances sylvicoles et technologiques de deux provenances de teck (Tectona grandis L. f) dans la forêt classée de la Lama. Thèse d'ingénieur agronome, Faculté des Sciences Agronomiques, Université d'AbomeyCalavi. $103 \mathrm{p}+$ annexes.

Blanchin J, Guillou J, Mompied D, Carreau JM. 2009. Les coupes d'amélioration en traitement régulier CRPF de Bretagne.

Bello AA. 2015. Estimation du stock de carbone de la biomasse aérienne des plantations de teck (Tectona grandis L. F.) de la forêt classée de la Lama (sud et centre Bénin). Mémoire de Master en Gestion des Ressources Naturelles et de la Biodiversité, Faculté des Sciences Agronomiques, Université d'AbomeyCalavi. $34 \mathrm{p}+$ annexes.

Bermejoa I, Canellas I, Miguel AS. 2003. Growth and yield models for teak plantations in Costa Rica Forest Ecology and Management. Www.elsevier.com/ locate/foreco.

Colin A, Lambert P. 2012. Analyse de la ressource forestière actuelle en Lorraine. Résultats statistiques et Représentations cartographiques. IGN : 12-0177. 45290 Nogent-sur Vernisson

Ganglo JC, Lejoly J, Pipar T, 1999. Le Teck (Tectona grandis L.f) au Bénin, gestion et perspectives. Bois et Forêts des Tropiques, 261(3): 17-27.

Ganglo JC, de Foucault B. 2006. Plant communities, forest site identification in Toffo reserve, South-Benin. Bois et Forêts des Tropiques, 288(2): 25-38 p.

Goreaud F, Pélissier R. 2000. Analyse de la structure spatiale de semis de points hétérogènes: exemples d'application à des peuplements forestiers, $49 \mathrm{p}$.

Gbesso FGH, Lougbégnon TO, Tenté BAH, Mensah GA, Sinsin BA. 2013. 
Caractérisations phytoécologique et structurale des groupements végétaux abritant Chrysophyllum albidum (G. Don) sur le plateau d'Allada au SudBenin. Afrique Science, 09(3): 147 158.

Goreaud F, Allain R, Courbaud B, Ngo Bieng MA, Pérot T, Piroche JN. 2007. Simuler des peuplements de structures variées pour faciliter l'utilisation des modèles "arbre" spatialisés Outils et méthodes, $131-161 \mathrm{p}$.

Herrero-Jáuregui C, García-Fernández C, Sist PL, Casado MA. 2012. Recruitment dynamics of two low-density neotropical multiple-use tree species. Plant Ecology, 212(9): 1501-1512.

Hitimana J, Kiyiapi JL, Njunge TJ. 2004. Forest Structure Characteristics in Disturbed and Undisturbed Sites of Mt. Elgon Moist Lower Montane Forest, Western Kenya. Department of Forestry, Moi University: Eldoret, Kenya.

Hochberg Y. 1988. A sharper Bonferroni procedure for multiple tests of significance. Biometrika 75, 800-803.

Koura K, Dissou EF, Ganglo JC. 2013. Caractérisation écologique et structurale des parcs à néré [Parkia biglobosa (Jacq.) R. Br. Ex G. Don] du département de la Donga au Nord-Ouest du Bénin. Int. J. Biol. Chem. Sci., 7(2): 726-738.

Kpadonou C. 2015. Caractéristiques structurales et dynamique des plantations forestières de teck (Tectona grandis L. f.) de la forêt classée d'Agrimey (Département du Zou, Bénin). Mémoire de Master en Aménagement et Gestion des Ressources Naturelles, Faculté des Sciences Agronomiques, Université d'Abomey-Calavi. $43 \mathrm{p}+$ annexes.

Massenet JY. 2010. Caractérisation et mesure des peuplements réguliers $25 \mathrm{p}$.
ONAB. 2005. Aménagement Participatif des Plantations Forestières d'Agrimey, Djigbé, Koto, Massi et Toffo: Période 2004-2023. ONAB: Bénin.

ONAB. 2014. Rapport des campagnes de reboisement 2011 - 2014. Direction Technique Bohicon. Bénin.

Pardé J, Bouchon J. 1988. Dendrométrie. ( $2^{\mathrm{e}}$ édn). Ecole Nationale de Génie Rural des Eaux et Forêts, ENGREF : Nancy, $328 \mathrm{p}$.

Pardé J. 2002. A ménagement et gestion. Le teck, en ses forêts et plantations tropicales Rev. For. Fr. LIV - 3-2002.

Rondeux J. 1999. La Mesure des Peuplements Forestiers. Presses Agronomiques de Gembloux, 522.

Sèwadé C. 2010. Dynamique des plantations de teck (Tectona grandis L.f.) gérées en régime de futaie dans les plantations forestières de la Lama au Sud-Bénin. Mémoire de DEA, FSA/UAC, Bénin, 103 p.

Sinsin FA. 2007. Etude comparative des caractéristiques sylvicoles et morphologiques de deux provenances de teck (Tectona grandis L.f.) dans les stations forestières de la lama. Mémoire pour l'obtention du diplôme d'ingénieur agronome, FSA/UAC. 92 p.

Toundoh P. 2013. Caractéristiques structurales, dynamique et productivité des plantations forestières de teck de la Lama (Sud et Centre Bénin). Mémoire de Master en Aménagement et Gestion des Ressources Naturelles, Faculté des Sciences Agronomiques, Université d'Abomey-Calavi. $30 \mathrm{p}+$ annexes.

Trainer J, Decleire Y. 2003. Résultats de l'aménagement des secteurs d'Agrimey, Djigbé, Toffo, Koto, Massi. ONAB, 26p. 